\title{
A Note on Stability Analysis of Taylor Collocation Method
}

\author{
Sinan Deniz ${ }^{1}$, Necdet Bildik²*, Mehmet Sezer ${ }^{3}$ \\ ${ }^{1}$ Manisa Celal Bayar University, Faculty of Science and Letters, Department of Mathematics, Muradiye Campus, \\ 45140,Manisa, Turkey, +90 2362013227 , \\ sinan.deniz@cbu.edu.tr \\ ${ }^{2}$ Manisa Celal Bayar University, Faculty of Science and Letters, Department of Mathematics, Muradiye Campus, \\ 45140,Manisa, Turkey, +90 2362013203 , \\ necdet.bildik@cbu.edu.tr \\ ${ }^{3}$ Manisa Celal Bayar University, Faculty of Science and Letters, Department of Mathematics, Muradiye Campus, \\ 45140,Manisa, Turkey, +90 236 2013202, \\ mehmet.sezer@cbu.edu.tr \\ ${ }^{*}$ Corresponding Author \\ Received: $16^{\text {th }}$ October 2016 \\ Accepted: $15^{\text {th }}$ November 2016 \\ DOI: http://dx.doi.org/10.18466/cbujos.302661
}

\begin{abstract}
In this study, we investigate the stability of Taylor collocation method for initial value problems in ordinary differential equations. Firstly, we try to show that Taylor collocation method for initial value problem is equivalent to a subset of the implicit Runge-Kutta methods. This equivalence enables us to prove that Taylor collocation method is absolutely stable (A-stable) for the considered equations.
\end{abstract}

Keywords - A-stability, ordinary differential equations, Taylor collocation method, Runge-Kutta methods, series solutions.

\section{Introduction}

There are many efficient analytical methods to solve nonlinear problems [1-5]. Taylor collocation method is one of them and its principle is simple: the solution of the considered differential equation is represented as a linear combination of known functions and the unknown coefficients in this representation are found by satisfying the associated conditions, and the differential equation at an appropriate number of points in the range of interest. In other words, it is basically a procedure for computing the coefficients in the Taylor expansion of the solution of differential equations. So far, this method has been applied to find the approximate solutions of many equations such as integral, difference, ordinary and partial differential equations [6-9]. Different types of this method have also been considered by many authors [10-12]. In [12], Lanczos discusses a form of this method which he calls the method of "selected points" using a polynomial or finite Chebyshev series to represent the solution and suggests using the zeros of a Chebyshev polynomial for the collocation points at which the differential equation is satisfied. Taylor collocation method shows the best advantage when the functions in the considered problem can be expanded to the Taylor series which converges rapidly. Otherwise, the accuracy of solution may not be good enough.

Stability analyses of some numerical methods have been studied by many authors [13, 14].There are a wide variety of forms of "stability" that have been studied in various contexts. One of them is zerostability which is the form of stability needed to guarantee convergence of a numerical method as the grid is refined ( $h \rightarrow 0$ ). In practice, we cannot compute this limit. Instead we usually perform a 
CBÜ F Bil. Dergi., Cilt 13, Sayı 1, 2017, 149-153 s

single calculation with some particular time step $h$. We need to choose the time step as large as possible to decrease the expense of the computation. However, we cannot estimate the size of $h$ required. So, we need another form of stability stronger than zero-stability: Absolute stability (A-stability). Astability is based on the linear test equation. However, it gives also useful information in determining an appropriate time step in nonlinear problems. In this study, we try to show that Taylor collocation method is A-stable by using the equivalence with Runge-Kutta methods.

\section{Taylor Collocation Method}

In this section, we briefly give the basic ideas of Taylor collocation method for solving initial value problem. Exact or approximate solution $y(x)$ of the initial value problem

$$
y^{\prime}=f(x, y), \quad y\left(x_{0}\right)=y_{0}, \quad a \leq c \leq b
$$

is considered in the matrix form by using Taylor polynomials:

$$
y(x) \cong \sum_{n=0}^{N} y_{n}(x-c)^{n}=X(x) Y,
$$

where

$$
\begin{aligned}
& y_{n}=\frac{y^{(n)}(c)}{n !} \\
& X(x)=\left[\begin{array}{llll}
1 & (x-c) & (x-c)^{2} \cdots & (x-c)^{N}
\end{array}\right], \\
& Y=\left[\begin{array}{llll}
y_{0} & y_{1} & \cdots & y_{N}
\end{array}\right]^{T} .
\end{aligned}
$$

So taking the derivative of Eq.(2), we have

$$
y^{\prime}(x)=X^{\prime}(x) Y=X(x) B Y
$$

where

$$
B=\left[\begin{array}{ccccc}
0 & 1 & 0 & \cdots & 0 \\
0 & 0 & 2 & \cdots & 0 \\
\vdots & \vdots & \vdots & \cdots & \vdots \\
0 & 0 & 0 & \cdots & N \\
0 & 0 & 0 & \cdots & 0
\end{array}\right] .
$$

Similar computations can be done for the function $f(x, y)$ to write it in the matrix form [15]. In order
CBU J. of Sci., Volume 13, Issue 1, 2017, p 149-153

to obtain the Taylor polynomial solution of equation (1) in the form (2), we compute the Taylor coefficients $y_{n}$ by means of the Taylor collocation points defined by

$$
x_{i}=a+\frac{b-a}{N} i, \quad i=0,1, \ldots, N .
$$

In order to get the best approximating solution of the equation, we need to take more terms from the Taylor expansion of functions; that is, the truncation limit $\mathrm{N}$ must be chosen to be large enough. But it should be emphasized that choosing $\mathrm{N}$ large reveals that the big augmentations of the dimensions of matrices in the solution process. For much more detail about the method of solution see $[6,15,16]$.

\section{Similarity with the Runge-Kutta Methods}

In this section, we show that implicit Runge-Kutta methods and Taylor collocation method have common procedure in the solution process. After showing these common procedures, we can discuss the stability of Taylor matrix method by using this similarity.

In Taylor collocation method, we define collocation points

$$
a=x_{0}<x_{1}<\cdots<x_{N}=b
$$

for the interval $[a, b]$. There are equal distances $h=\frac{b-a}{N}$ between all collocation points. Starting from the initial value $x_{0}=a$, we proceed by using all collocation points until the end of the interval or to the last collocation point $x_{N}=b$. After applying the method of solution $[15,16]$, Taylor collocation method for the initial value problem (1) can be considered as:

$$
\begin{gathered}
y\left(x_{0}\right)=y_{0} \\
y^{\prime}\left(x_{i}\right)=f\left(x_{i}, y\left(x_{i}\right)\right), \quad i=1, \ldots, N .
\end{gathered}
$$


CBÜ F Bil. Dergi., Cilt 13, Sayı 1, 2017, 149-153 s

For the initial value problem (1), we are actually searching for an approximation to the solution at $x=x_{0}+h$. In order to show the similarity with Runge-Kutta methods, equations in (8) may be written equivalently:

$$
\begin{gathered}
y\left(x_{0}\right)=y_{0} \\
y^{\prime}\left(x_{0}+\alpha_{i} h\right)=f\left(x_{0}+\alpha_{i} h, y\left(x_{0}+\alpha_{i} h\right)\right), \quad i=1, \ldots, N
\end{gathered}
$$

where $\alpha_{1}, \ldots, \alpha_{N}$ are real numbers. Taylor coefficients $y_{n}$ in (3) arise after solving the algebraic equations which come from the solution process $[15,16]$. They are also obtained by satisfying the differential equation at the points $x=\alpha_{i} h$. Letting $c=0$ in (2) :

$$
y_{n}(x) \cong \sum_{n=0}^{N} y_{n} x^{n}
$$

and substituting this approximate solution into the Eq.(1) yields:

$$
\sum_{n=1}^{N} n y_{n} h^{n-1} \alpha_{i}^{n-1}=f\left(h \alpha_{i}, \sum_{n=0}^{N} y_{n} h^{n} \alpha_{i}^{n}\right), \quad i=1, \ldots, N
$$

Let us define

$$
f_{i}=f\left(h \alpha_{i}, \sum_{n=0}^{N} y_{n} h^{n} \alpha_{i}^{n}\right)
$$

Considering (11) and (12), we can deduce that $y_{n}(x)$ and the coefficients $y_{n}$ can be written as the linear combinations of $f_{i}$; that is, the method takes the form of an $N$ - stage implicit Runge-Kutta method in the form

$$
\begin{aligned}
& k_{i}=f\left(x_{0}+\alpha_{i} h, y_{0}+h \sum_{j=1}^{N} a_{i j} k_{j}\right) \\
& y_{1}=y_{0}+h \sum_{i=1}^{N} b_{i} k_{i}
\end{aligned}
$$

CBU J. of Sci., Volume 13, Issue 1, 2017, p 149-153

where $a_{i j}, b_{i}(i, j=1, \ldots, N)$ are real numbers and $\alpha_{i}=\sum_{j=1}^{i-1} a_{i j}[17]$.

It is clear that Runge-Kutta methods work in terms of values of the functions whereas Taylor collocation method deals with polynomial coefficients. In fact this is the only difference between these two methods. As in Taylor collocation method, $\mathrm{N}$ must be also chosen to be large enough in Runge-Kutta methods to get better results.

\section{A-Stable Taylor Collocation Method for Initial Value Problem}

In Section 3, we have shown that Taylor collocation method for initial value problem is equivalent to a sub-set of the implicit Runge-Kutta methods, in the sense that the final values at the end of the step are algebraically the same. In this section, we show that Taylor collocation method is absolutely stable (Astable) by using this equivalence. It should be noted that the stability of a numerical method depends on the problem which it is applied to and the study of absolute stability requires consideration only of equations with constant coefficients. So, we use the model problem

$$
y^{\prime}=q y
$$

where $q$ is a complex constant.

Stability of Runge-Kutta methods have been discussed by many authors [12-14]. In [15], Dahlquist has defined A-stability for the equation (14) in his own efforts. He established that 4th order RungeKutta process is not A-stable. It is also known that, neither explicit finite-difference method nor implicit method of order higher than 2 is A-stable [16]. So, we need to consider implicit Runge-Kutta method of order less than 3 to show that Taylor collocation method is A-stable or to obtain the region of Astability. The simplest example of an implicit Runge-Kutta method is the backward Euler method:

$$
Y_{i+1}=Y_{i}+h f\left(x_{i+1}, Y_{i+1}\right), \quad i=1, \ldots, N
$$

Applying (15) to the model equation (14) yields: 


$$
Y_{n+1}=Y_{n}+h q Y_{n+1} \Rightarrow Y_{n}=\frac{Y_{0}}{(1-h q)^{n}}
$$

The numerical solution (16) will decrease only if

$$
\left|\frac{1}{(1-h q)^{n}}\right| \leq 1 \Rightarrow\left|\frac{1}{1-h q}\right| \leq 1 \Rightarrow|1-h q| \geq 1
$$

then

$$
h \geq \frac{2}{|q|}
$$

From (18), we can say that Taylor collocation method is A-stable when $h \geq \frac{2}{|q|}$, otherwise it is unstable. Note that there are two parameters $h$ and $q$, but only their product $z=h q$ matters.

With this known, one can also obtain the stability region by sketching the graph of the inequality (17):

$$
\left(1-h q_{r}\right)^{2}+\left(h q_{i}\right)^{2} \geq 1
$$

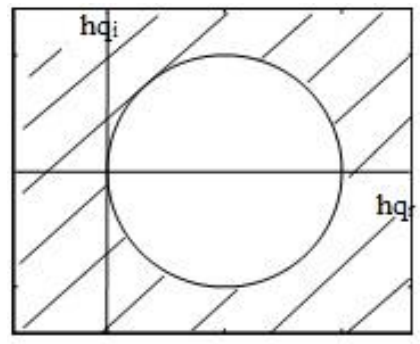

Fig.1: Stability region for Taylor collocation method

\section{Examples}

In this section, we take linear and nonlinear first order initial value problems to check the A-stability region of Taylor collocation method. We should remind that one can also perform the stability analysis for nonlinear problems by knowing that the model equation (14) is practically the local linear approximation (also known as a linearization) of the considered problems (1). In other words, the equation (14) is relevant for predicting stability of solutions of the nonlinear ordinary differential equations which can be generated from (1).

Ex 1. Consider the simple linear equation

$$
y^{\prime}=-100 y, \quad 0 \leq x \leq 1
$$

with any initial condition. In order to guarantee that the round-off and truncation errors will decay, we need to choose $h \geq \frac{2}{100}=0,02$ to solve the problem (20). In other words, the truncation limit $N$ in the approximate solution (2) must be taken as $N \leq 50$.

Ex 2. Let us consider the Riccati differential equation

$$
y^{\prime}=x^{2}+y^{2}, \quad y(0)=1, \quad 0 \leq x \leq 1
$$

which have no exact solution. This problem is solved in [17] by using Taylor collocation method. To examine the stability, we first need to linearize the equation as:

$$
y^{\prime} \approx f_{y}(x, y)=2 y \text {. }
$$

Now we can say that, one needs to take $h \geq \frac{2}{2}=1$ or $N=1$ to make the Taylor collocation method applied to (21) is A-stable. Choosing $N \geq 1$ will cause to increase the round-off and truncation errors. However, $N=1$ is too small to obtain satisfactory approximate solution. Therefore, one needs to take the extended interval to gain the A-stability and better results for Taylor collocation method.

\section{Discussion}

In this paper, we examine the stability of Taylor collocation method. Our results show that Taylor collocation method for initial value problem is equivalent to a subset of the implicit Runge-Kutta methods. By using the some familiar information about Runge-Kutta methods, we prove that Taylor collocation method is absolutely stable for the model problem. We also take different type of ex- 
CBÜ F Bil. Dergi., Cilt 13, Sayı 1, 2017, 149-153 s

amples to illustrate the cases. In the nonlinear case, we emphasize that one needs to linearize the equation to investigate the stability. It is worth mentioning that these results can also be expanded to the higher order functions by following the same procedure.

\section{References}

[1] Bildik, N.; Deniz, S. Implementation of taylor collocation and adomian decomposition method for systems of ordinary differential equations. Proceedings of the International Conference on Numerical Analysis and Applied Mathematics 2014. AIP Publishing. 2015; 1648 (1).

[2] Bildik, N.; Deniz, S. Applications of Taylor collocation method and Lambert $\mathrm{W}$ function to systems of delay differential equations. Turk. J. Math. Comput. Sci., Article ID 20130033. 2013; 1-13.

[3] Bildik, N.; Deniz, S. Comparative study between Optimal Homotopy Asymptotic Method and PerturbationIteration Technique for different types of nonlinear equations. Iranian Journal of Science and Technology Transactions A: Science. 1-8, (2016); doi: 10.1007/s40995-016-00392.

[4] Bildik, N.; Deniz, S. The use of Sumudu Decomposition Method for solving Predator-Prey Systems. Mathematical Sciences Letters.2016; 5(3), 285-289.

[5] Deniz, S.; Bildik, N. Optimal Perturbation Iteration Method for Bratu-Type Problems. Journal of King Saud University - Science (2016). (In press) http://dx.doi.org/10.1016/j.jksus.2016.09.001.

[6] Karamete, A.; Sezer,M. A Taylor collocation method for the solution of linear integro-differential equations . International Journal of Computer Mathematics. 2002; 79(9), 987-1000.

[7] Gülsu, M.; Öztürk,Y.; Sezer,M. A new collocation method for solution of mixed linear integro-differentialdifference equations. Applied Mathematics and Computation . 2010; 216(7), 2183-2198.

[8] Bildik, N.; Deniz, S. Comparison of solutions of systems of delay differential equations using Taylor collocation method, Lambert $\mathrm{W}$ function and variational iteration method. Scientia Iranica. Transaction D, Computer Science \& Engineering and Electrical Engineering. 2015; 22(3), 1052-1058.

[9] Deniz, S.; Bildik, N. Comparison of Adomian Decomposition Method and Taylor Matrix Method in Solving Different Kinds of Partial Differential Equations.
CBU J. of Sci., Volume 13, Issue 1, 2017, p 149-153

International Journal of Modeling and Optimization. 2014; 4(4), 292-298.

[10] Vasilyev, O.; Bowman, C. Second-generation wavelet collocation method for the solution of partial differential equations. Journal of Computational Physics. 2000; 165 (2), 660-693.

[11] Russell-Robert D.; Lawerence, F. A collocation method for boundary value problems. Numerische Mathematik. 1972; 19(1), 1-28.

[12] Lanczos, C. Trigonometric interpolation of empirical and analytical functions. Journal of Mathematics and Physics. 1938; 17(1), 123-199.

[13] Klaus-Jürgen,B.; Wilson,E. Numerical methods in finite element analysis. Press: Prentince-Hall Inc., Berkeley, 1976; 552 pp.

[14] Kloeden-Peter, E.; Eckhard, P. Higher-order implicit strong numerical schemes for stochastic differential equations. Journal of statistical physics. 1992; 66 (1-2), 283-314.

[15] Gökmen, E.; Sezer, M. Taylor collocation method for systems of high-order linear differential-difference equations with variable coefficients. Ain Shams Engineering Journal. 2013; 4(1), 117-125.

[16] Sezer, M. A method for the approximate solution of the second-order linear differential equations in terms of Taylor polynomials. International Journal of Mathematical Education in Science and Technology. 1996; 27(6), 821-834.

[17] Butcher, J.C. The numerical analysis of ordinary differential equations: Runge-Kutta and general linear methods. Wiley-Interscience. 1987.

[18] Wright, K. Some relationships between implicit Runge-Kutta, collocation and Lanczos $\tau$ methods, and their stability properties. BIT Numerical Mathematics. $1970 ; 10(2)$ 217-227.

[19] Dekker, K.; Verwer, J.G. Stability of Runge-Kutta methods for stiff nonlinear differential equations. Vol. 984. Amsterdam: North-Holland. 1984.

[20] Dahlquist, G. Generalized disks of contractivity for explicit and implicit Runge-Kutta methods. No. TRITANA-79-06. CM-P00069451. 1979.

[21] Hairer, E.; Nørsett, S.P.; Wanner,G. Solving ordinary differential equations I. Nonstiff problems. 1987.

[22] Gülsu, M.; Sezer, M. On the solution of the Riccati equation by the Taylor matrix method. Applied Mathematics and Computation. 2006; 176(2), 414-421. 